\title{
The Differences of Interstitial Lung Diseases in High-Resolution Computerized Tomography and Pulmonary Function Test among Different Connective Tissue Diseases, and the Correlated Factors
}

\author{
Zhen Jiang1, Wenyou Pan', Jinhui Tao², Xiangpei Li ${ }^{2 *}$ \\ ${ }^{1}$ Department of Rheumatology and Immunology, The Affiliated Huai'an No.1 People's Hospital of Nanjing Medical University, \\ Huai'an, China \\ ${ }^{2}$ Department of Rheumatology and Immunology, Anhui Provincial Hospital, Anhui Medical University, Hefei, China \\ Email: *lixiang pei55@126.com
}

How to cite this paper: Jiang, Z., Pan, W.Y., Tao, J.H. and Li, X.P. (2018) The Differences of Interstitial Lung Diseases in High-Resolution Computerized Tomography and Pulmonary Function Test among Different Connective Tissue Diseases, and the Correlated Factors. Open Journal of Rheumatology and Autoimmune Diseases, 8, 53-65.

https://doi.org/10.4236/ojra.2018.82005

Received: March 24, 2018

Accepted: May 18, 2018

Published: May 21, 2018

Copyright $\odot 2018$ by authors and Scientific Research Publishing Inc. This work is licensed under the Creative Commons Attribution International License (CC BY 4.0).

http://creativecommons.org/licenses/by/4.0/

\begin{abstract}
Objective. To study the difference of interstitial lung diseases (ILDs) in high-resolution computerized tomography and pulmonary function test among different connective tissue diseases (CTDs). Methods. 209 patients with different CTDs were recruited and underwent lung HRCT and PFT. Eerythrocyte sedimentation rate (ESR), C-reactive protein (CRP), serum ferritin (SF), anti-SSA, and so on were tested. Based on HRCT, a patient was classified into ILD group (CTD+ILD) or non-ILD group (CTD-ILD). HRCT, PFT, and laboratory markers were compared according to CTDs and CTD-associated ILDs. Results. The incidences of ILD were 79.6\%, 82.0\%, $89.7 \%$, and $97.1 \%$ respectively for Rheumatoid arthritis (RA), primary Sjogren's symptom (pSS), dermatomyositis/polymyositis (DM/PM), and systemic sclerosis (SSc) groups. RA and pSS patients exhibited more nodules, patching, ground-glass opacity, and cord shadow foci in HRCT, DM/PM and SSc patients exhibited more reticular opacity and honeycombing foci. RA and pSS patients exhibited more obstructive ventilatory disorder, small airway dysfunction and emphysema in PFT, and DM/PM and SSc patients exhibited more restrictive ventilatory disorder, mixed ventilatory disorder. ESR, CRP and SF were significantly higher in total CTD+ILD group than in total CTD-ILD group ( $P=0.047,0.006,0.004$, respectively), and higher in different CTD+ ILD groups than in comparable CTD-ILD groups $(P=0.049,0.048$, and 0.023 , pSS+ILD, SSc+ILD and RA+ILD compared to pSS-ILD, SSc-ILD
\end{abstract}


and RA-ILD, respectively for ESR, CRP, SF). The positive rate of anti-SSA was significantly higher in DM/PM+ILD group than in DM/PM-ILD group $(P=$ 0.025). Conclusions. The manifestations and incidences of ILDs differ among different CTDs in HRCT and PFT, and inflammation and anti-SSA are positively correlated with ILDs in different CTDs, which provide important evidences for judging disease condition and prognosis.

\section{Keywords}

Connective Tissue Diseases, Interstitial lung Disease, High-Resolution Computerized Tomography, Pulmonary Function Test, Inflammation

\section{Introduction}

Lung is one of main involved organs in diffuse connective tissue diseases (CTDs). As the use of high-resolution computerized tomography (HRCT) and pulmonary function tests (PFT) has become more frequent in clinics, CTDs, such as rheumatoid arthritis (RA), primary Sjogren's symptom (pSS), dermatomyositis/polymyositis (DM/PM), and systemic sclerosis (SSc) have increasingly been found with interstitial lung disease (ILD) [1]. However, little is known about how the manifestations of lung HRCT and PFT vary among different ILDs associated with those CTDs (CTD+ILD) and what factors are related to those ILDs.

Inflammation is involved in the pathogenesis of many autoimmune diseases. C-reactive protein (CRP), erythrocyte sedimentation rate (ESR), and serum ferritin (SF) levels are higher in patients with CTD+ILD than patients with CTD but without ILD (CTD-ILD) [2] [3] [4]. These changes have been associated with the prognosis of patients with CTD+LD [2] [3] [4]. Several auto-antibodies are also reported associated with inflammatory myopathy-associated ILD [5] [6]. In current study, we compared the incidences and the differences of ILDs in HRCT and PFT among patients with different CTDs, and analyzed the correlations between ILDs and changes in inflammatory markers and auto-antibody levels.

\section{Materials and Methods}

\subsection{Patients}

The consecutive inpatients and outpatients (adults) with RA, pSS, DM/PM, and SSc were recruited from Department of Rheumatology and Immunology, Anhui Provincial Hospital, Hefei, China from January to September of 2013. The diagnosis of those diseases was based on the 2009 European League Against Rheumatism (EULAR) criteria for RA, the 2002 international classification criteria for pSS, the Bohan and peter criteria for DM/PM, and the 1980 American College of 
Rheumatology (ACR) criteria for SSc, The inclusion criteria were described in our published article [7]. Patients with pulmonary infection, tumours, or tuberculosis were excluded. The diagnosis of ILD was basis on the definition on HRCT from the Nomenclature Commitee of the Fleischner Society [8]. The paper was examined and approved to publish by the ethics committee of Anhui Provincial Hospital (Supplement-Ethics document).

\subsection{Experimental Data and Treatment}

Age, disease duration, inflammatory markers (ESR, CRP, and SF) and auto-antibodies (antinuclear antibody (ANA, indirect immunofluorescence), anti-SSA (immunoblotting), anti-endothelial cell antibody (AECA, ELISA)), and the previous three-month treatment protocols were collected. Based on the used drugs, the treatment schemes were classified into six levels $(1,2,3,4,5$, and 6 , indicating symptomatic treatment, only glucocorticoids used, one kind of disease modified anti-rheumatic drug (DMARD) used, glucocorticoid and one DMARD used, two DMARDs used, glucocorticoid and two or more DMARDs used, respectively). Because of lower positive rate and the relatively smaller sample, anti-JO-1 and anti-SCL-70 were not included in our study.

All patients were evaluated by chest HRCT and PFT. The devices were described in our article [7]. An independent radiologist read the films, and an expert in PFT made the diagnosis of pulmonary function.

\subsection{Group of Patients and Analyze}

Based on HRCT, a patient was classified into ILD group (CTD+ILD) or non-ILD group (CTD-ILD). Every kind of CTD patients and all patients were classified into CTD+ILD groups and comparable CTD-ILD groups respectively. We compared the incidences of ILDs and the frequencies of HRCT and PFT manifestations among four CTDs. The levels or positive rates of inflammatory markers and auto-antibodies were compared among different CTDs, and between different CTD+ILD groups and comparable CTD-ILD groups. We also analyzed the distributions of confounders (age, disease duration, and treatment protocol) among different CTD groups, and between CTD+ILD groups and comparable CTD-ILD groups.

\subsection{Statistical Analysis}

Quantitative data were expressed as means \pm SD (standard deviation) or medians and interquartile ranges (IQR), and compared by one-way ANOVA or an independent-sample T test, or Kruskal-Wallis test. Qualitative data were compared by $\chi^{2}$ test. Multiple comparisons were estimated by S-N-K (Student Newman Keuls) or Mann-Whitney U test. The software SPSS 10.01 was used. Differences with $P$ values $<0.05$ were considered significantly. When multiple comparisons were used, the overall experiment-wise error rate was set at $\alpha=$ 0.05 . 


\section{Results}

\subsection{Age and Disease Duration}

209 patients (30 men and 179 women) were included into current study, which included 52 RA (4 men and 48 women), 57 pSS (1 man and 56 women), 59 DM/PM (23 men and 36 women), and 41 SSc patients ( 2 men and 39 women). The mean age of the patients in the total, RA, pSS, DM/PM, and SSc groups were $46.8 \pm 14.6,51.8 \pm 13.6,48.3 \pm 13.4,43.6 \pm 15.5$, and $43.1 \pm 14.6$ years, respectively, and that in RA group was significantly higher than those in DM/PM and SSc groups $(P=0.002,0.003$, respectively). The mean ages in pSS+ILD, $\mathrm{DM} / \mathrm{PM}+\mathrm{ILD}$ and total CTD+ILD groups were significantly higher than those in comparable CTD-ILD groups ( $P=0.003,0.039,0.002$, respectively). The median disease duration in total, RA, pSS, DM/PM, and SSc groups was 48 (12 - 96), 84 (12.0 - 162.0), 54 (24.0 - 114.0), 12 (4.8 - 51.0), and 48 (24.0 - 87.0) months, respectively. The median disease duration was significantly less in DM/PM group than in pSS, RA, and SSc groups, and was longest in RA group, however, it did not significantly differ between various CTD-ILD groups and comparable CTD+ILD groups (Table S1 and Table S2).

\subsection{Treatment Protocols}

The accumulated points of the treatment protocols did not significantly differ among four CTD groups, and among total CTD+ILD group and comparable total CTD-ILD group $(P>0.05$, data not shown). The DMARDs are shown in Table S3.

\subsection{High-Resolution Computerized Tomography (HRCT)}

196 patients underwent chest HRCTs, which included 49 RA (92.5\%), 55 pSS (91.7\%), $58 \mathrm{DM} / \mathrm{PM}$ (98.3\%), and $34 \mathrm{SSc}(73.2 \%)$ patients. Based on HRCT, there were 163 patients $(83.2 \%, 23$ men $(14.1 \%)$ and 140 women $(85.9 \%)$ involved) in CTD+ILD group, and 33 patients (16.8\%, 5 men (15.2\%) and 28 women (84.8\%) involved) in CTD-ILD group. The difference of the ratio of genders was significant between two groups ( $P=0.024, \chi^{2}$ test), and there were more men in CTD-ILD group than that in CTD+ILD group. The incidences of ILD were $79.6 \%$ (39), 82.0\% (45), 89.7\% (52), and 97.1\% (33), respectively for RA, pSS, DM/PM and SSc groups, and the difference was not significant ( $P=$ 0.051, data not shown).

There were seven types of HRCT: reticular opacity or honeycombing, cord shadow, patching or ground-glass opacity, nodule, emphysema or bulla or bronchiectasis, slight inflammation, and normal HRCT. The percentages of normal HRCTs were significantly higher in pSS and RA groups than in SSc group. Nodule frequencies decreased in the following order: pSS, RA, DM/PM, and SSc, meanwhile, SSc group presented a statistically lower frequency $(P=$ 0.029 and 0.037 compared to pSS and RA, respectively). Reticular opacity and honeycombing frequencies increased in the following order: pSS, RA, DM/PM 
and SSc, meanwhile, SSc group presented a statistically higher frequency $(P=$ 0.002 and 0.005 compared to pSS and RA, respectively). The frequencies of the other manifestations, such as cord shadow, patching or ground-glass opacity, emphysema, bulla, bronchiectasis, slight inflammation, and combined manifestations (patching, ground-glass opacity and slight inflammation), did not significantly differ among four CTD groups although patching or ground-glass opacity and cord shadow decreased in the following order: pSS, RA, DM/PM and SSc. The data are shown in Table 1.

\subsection{Pulmonary Function Test (PFT)}

PFT was obtained from 41 pSS (71.9\%), 38 RA (73.1\%), 48 DM/PM (81.4\%), and 30 SSc (73.2\%) patients. PFT manifestations were also classified into 7 types: obstructive ventilatory disorder (OVD), restrictive ventilatory disorder (RVD), mixed ventilatory disorder (MVD), small airway dysfunction, emphysema, diffusion function impairment (DFI), and normal PFT based on the associated parameters. DFI was the most common manifestation in four CTD groups. The data are shown in Table 2.

The frequency of normal PFT did not significantly differ among four CTD groups. Small airway dysfunction decreased in the following order: pSS, RA, SSc and DM/PM although the difference was not significant. The frequency of emphysema decreased in the following order: pSS, RA, DM/PM, and SSc although the difference was not significant. The frequency of OVD decreased in the following order: pSS, RA, SSc and DM/PM, meanwhile, DM/PM presented a significantly lower frequency $(P=0.008$ and 0.014 compared to pSS and RA,

Table 1. Comparison of the manifestations of lung HRCT among different connective tissue diseases.

\begin{tabular}{cccccc}
\hline HRCT & pSS (55) & RA (49) & DM/PM (58) & SSc (34) & $P$ \\
\hline Normal HRCT & $10(18.0)$ & $10(20.4)$ & $6(10.3)$ & $1(2.9)$ & 0.023 \\
Reticular opacity or honeycombing & $10(10.9)$ & $10(20.4)$ & $18(31.0)$ & $17(50.0)$ & 0.006 \\
Nodule & $7(12.7)$ & $6(12.2)$ & $4(6.9)$ & $0(0)$ & 0.043 \\
Cord shadow & $12(21.8)$ & $9(18.4)$ & $11(19.0)$ & $4(11.8)$ & 0.696 \\
$\begin{array}{c}\text { Patching or ground-glass opacity } \\
\text { Emphysema or bulla or bronchiectasis }\end{array}$ & $16(29.1)$ & $16(22.4)$ & $12(20.7)$ & $6(17.6)$ & 0.287 \\
$\begin{array}{c}\text { Slight inflammation } \\
\text { Patching, ground-glass opacity, and } \\
\text { slight inflammation }\end{array}$ & $4(7.3)$ & $4(8.2)$ & $2(3.4)$ & $5(14.7)$ & 0.290 \\
\hline
\end{tabular}

Data are presented as number and percentage (No/\%). Normal HRCT: The percentage in SSc group was significantly lower than those in pSS and RA groups ( $P=0.02$ and 0.012 , respectively). Reticular opacity or honeycombing: The percentage in SSc group was significantly higher than those in pSS and RA groups ( $P=0.002$ and 0.005 , respectively). Nodule: The percentage in SSc group was significantly lower than those in pSS and RA groups ( $P=0.029$ and 0.037 , respectively). Abbreviations: HRCT: High-resolution computerized tomography, pSS: primary Sjogren's symptom, RA: rheumatoid arthritis, DM/PM: dermatomyositis/polymyositis, SSc: systemic sclerosis. 
Table 2. Comparison of the manifestations of PFT among different connective tissue diseases.

\begin{tabular}{cccccc}
\hline PFT & pSS (41) & RA (38) & DM/PM (48) & SSc (30) & $P$ \\
\hline OVD & $6(14.6)$ & $5(13.2)$ & 0 & $1(3.3)$ & 0.007 \\
RVD & $6(14.6)$ & $8(21.1)$ & $13(27.1)$ & $11(36.7)$ & 0.171 \\
MVD & $9(22.0)$ & $6(15.8)$ & $15(31.3)$ & $14(46.7)$ & 0.029 \\
Small airway dysfunction & $5(12.2)$ & $3(7.9)$ & $1(2.1)$ & $1(3.3)$ & 0.209 \\
Emphysema & $7(17.1)$ & $6(15.8)$ & $6(12.5)$ & $3(10.0)$ & 0.818 \\
DFI & $33(80.5)$ & $26(68.4)$ & $26(54.2)$ & $25(83.3)$ & 0.015 \\
RVD plus MVD & $15(36.6)$ & $14(36.8)$ & $22(45.8)$ & $22(73.3)$ & 0.008 \\
OVD plus MVD & $11(26.8)$ & $9(23.7)$ & $15(31.3)$ & $14(46.7)$ & 0.195 \\
\hline
\end{tabular}

Data are presented as number and percentage (No/\%). OVD: The percentage in DM/PM group was statistically lower than those in pSS and RA groups ( $P=0.008$ and 0.014 , respectively). MVD: The percentage in SSc group was statistically higher than those in pSS and RA groups ( $P=0.028$ and 0.006 , respectively). RVD plus MVD: The percentage in DM/PM group was significantly higher than those in pSS, RA and $\mathrm{DM} / \mathrm{PM}$ groups $(P=0.002,0.003$, and 0.017 , respectively). DFI: The percentage in DM/PM group was statistically lower than those in SSc and pSS groups $(P=0.008$ and 0.009 , respectively). Abbreviations: PFT: Pulmonary function test; OVD: obstructive ventilatory disorder; RVD: restrictive ventilatory disorder; MVD: mixed ventilatory disorder; DFI: diffusion function impairment; other abbreviations are as in Table 1.

respectively). The frequency of RVD increased in the following order: pSS, RA, $\mathrm{DM} / \mathrm{PM}$, and SSc although the difference was not significant. The frequency of MVD increased in the following order: RA, pSS, DM/PM and SSc, meanwhile, SSc group presented a significantly higher frequency $(P=0.028$ and $0.006 \mathrm{com}-$ pared to pSS and RA, respectively). The frequency of RVD plus MVD increased in the following order: pSS, RA, DM/PM and SSc; meanwhile, SSc group presented a significantly higher frequency $(P=0.002,0.003$, and 0.017 compared to pSS, RA, and DM/PM, respectively). The frequency of OVD plus MVD did not significantly differ among four CTD groups. PSS and SSc groups presented significantly higher frequencies for DFI ( $P=0.009$ and 0.008 , respectively, compared to $\mathrm{DM} / \mathrm{PM})$. In a word, there were more small airway dysfunction, emphysema and OVD in pSS and RA groups compared with DM/PM and SSc groups, and more RVD and MVD in DM/PM and SSc groups compared with pSS and RA groups. The data are shown in Table 2.

\subsection{Auto-Antibodies}

The titres of ANA in pSS and SSc groups were significantly higher than in RA and DM/PM groups. The frequency of anti-SSA was significantly higher in pSS, $\mathrm{DM} / \mathrm{PM}$, and SSc than in RA. The frequency of AECA did not significantly differ among four CTD groups $(P=0.149)$. Data are shown in Table 3 . The distributions of these antibodies did not significantly differ between total CTD-ILD group and total CTD+ILD group, and between the various CTD-ILD groups and comparable CTD+ILD groups (data not shown) except that the positive rate of 
Table 3. Comparison of the positive rates (\%) and titres for auto-antibodies among different connective tissue diseases.

\begin{tabular}{cccccc}
\hline & pSS & RA & DM/PM & SSc & $P$ \\
\hline ANA & $3200(0-32,000)$ & $100(0-10,000)$ & $100(0-10,000)$ & $10,000(320-32,000)$ & 0.000 \\
Anti-SSA & $71.4(40 / 56)$ & $12.2(6 / 49)$ & $22.2(12 / 54)$ & $34.9(15 / 43)$ & 0.000 \\
AECA & $8.6(3 / 35)$ & $10.6(5 / 47)$ & $19.5(8 / 41)$ & $3.2(1 / 31)$ & 0.149 \\
\hline
\end{tabular}

ANA titres were transformed into reciprocal values. The data are presented as medians and IQR for ANA and as percentage for other antibodies. The numbers in round brackets after percentages represent the numbers of patients with comparable positive antibodies and the numbers of all patients who were tested by comparable antibodies. The numbers of patients who underwent testing ANA were 56, 49, 54, and 43 respectively. ANA: The medians of pSS and SSc groups were significantly higher than those of RA and DM/PM groups $(P=0.000,0.000,0.000$, and 0.000$)$. Anti-SSA: The positive rate in RA group was significantly lower than those in pSS, DM/PM and SSc groups ( $P=0.000,0.00$, and 0.000 , respectively). Abbreviations: ANA: antinuclear antibody, AECA: anti-endothelial cell antibody; other abbreviations are as in Table 1.

anti-SSA in DM/PM+ILD group was significantly higher than that in DM/PM-ILD group $(P=0.025$, Table 4$)$.

\subsection{Inflammatory Markers}

The medians of ESR and CRP in RA group were significantly higher $(P=0.003$, $0.000,0.000$ compared to $\mathrm{pSS}, \mathrm{DM} / \mathrm{PM}$, and SSc, respectively (ESR), and $P=$ $0.000,0.002,0.001$ compared to $\mathrm{pSS}, \mathrm{DM} / \mathrm{PM}$, and SSc, respectively (CRP)). The median of SF in DM/PM group was significantly higher $(P=0.004,0.002$, and 0.002 compared to pSS, RA, and SSc, respectively). The data are shown in Table 5. ESR, CRP, and SF were all significantly higher in total CTD+ILD group than in total CTD-ILD groups (Table 6). Finally, ESR, CRP, and SF (except for SSc) were higher in various CTD+ILD groups than in comparable CTD-ILD groups, meanwhile, ESR was significantly higher in pSS+ILD, CRP was significantly higher in SSc+ILD, and SF was significantly higher in RA+ILD ( $P=0.049,0.048$, and 0.023 , compared to pSS-ILD, SSc-ILD and RA-ILD, respectively, data not shown).

\section{Discussion}

CTD+ILD is a leading cause of death in CTD patients [9] [10]. However, little is known about differences in the incidences and manifestations of ILD among different CTDs (based on lung HRCT and PFT evaluations). In current study, the manifestations of ILD were different depending on different CTDs in HRCT and PFT although the difference of the incidences was not significant (may be due to the smaller sample because the $P$ value (0.051) was close to 0.05$)$. pSS and RA groups had the lower incidences and mild condition, and DM/PM plus SSc groups had the higher incidences and severe condition. Moreover, ESR, CRP, SF, and anti-SSA were related to ILD associated with comparable CTDs.

HRCT is useful for diagnosis of ILD and monitor of the condition of ILD. In current study, more normal lung HRCTs, nodules, patching, ground-glass 
Table 4. Comparison of positive rates of anti-SSA between different CTD-ILD groups and comparable CTD+ILD groups.

\begin{tabular}{ccccc}
\hline & pSS $(56)$ & RA $(49)$ & DM/PM $(54)$ & SSc $(41)$ \\
\hline CTD+ILD & $80.6(25 / 31)$ & $8.8(3 / 34)$ & $26.7(12 / 45)$ & $33.3(12 / 36)$ \\
CTD-ILD & $60.0(15 / 25)$ & $20.0(3 / 15)$ & $0(0 / 9)$ & $40.0(2 / 5)$ \\
$P$ & 0.089 & 0.531 & 0.025 & 0.771 \\
\hline
\end{tabular}

Data are presented as \% (No). The numbers in round brackets after percentages represent the numbers of patients with positive anti-SSA and the numbers of all patients in every CTD+ILD or CTD-ILD group. Abbreviations: CTD-ILD: connective tissue disease without interstitial lung disease, CTD+ILD: CTD with ILD, other as in Table 1.

Table 5. Comparison of ESR, CRP, and SF among different connective tissue diseases.

\begin{tabular}{lccccc}
\hline & $\mathrm{pSS}(57)$ & $\mathrm{RA}(52)$ & $\mathrm{DM} / \mathrm{PM}(59)$ & $\mathrm{SSc}(41)$ & $P$ \\
\hline ESR $(\mathrm{mm} / \mathrm{h})$ & $22.0(3.0-126.8)$ & $51.0(8.3-124.4)$ & $15.5(3.0-104.2)$ & $20.0(2.1-99.6)$ & 0.000 \\
$\mathrm{CRP}(\mathrm{mg} / \mathrm{L})$ & $3.3(3.2-45.2)$ & $22.7(3.2-174.4)$ & $4.9(3.2-75.6)$ & $4.2(3.1-97.1)$ & 0.000 \\
$\mathrm{SF}(\mathrm{ng} / \mathrm{mL})$ & $112.0(7.4-908.3)$ & $107.8(7.2-852.5)$ & $209.1(23.6-1723.0)$ & $92.8(24.8-567.7)$ & 0.002 \\
\hline
\end{tabular}

Data are presented as medians and IQR. ESR: The median of RA group was significantly higher than those of pSS, DM/PM, and SSc groups ( $P=0.003,0.000$, and 0.000 , respectively). CRP: The median of RA group was significantly higher than those of pSS, DM/PM, and SSc groups $(P=0.000,0.002$, and 0.001 , respectively). SF: The median of DM/PM group was significantly higher than those of pSS, RA, and SSc groups ( $P$ $=0.004,0.002$, and 0.002, respectively). Abbreviations: ESR: erythrocyte sedimentation rate, CRP: C-reactive protein, SF: serum ferritin. Other abbreviations are as in Table 1.

Table 6. Comparison of the ESR, CRP, and SF between total CTD-ILD and comparable CTD+ILD groups.

\begin{tabular}{cccc}
\hline & ESR $(\mathrm{mm} / \mathrm{h})$ & CRP $(\mathrm{mg} / \mathrm{L})$ & $\mathrm{SF}(\mathrm{ng} / \mathrm{mL})$ \\
\hline CTD-ILD (27) & $19.0(9.0-43.5)$ & $3.3(3.2-9.0)$ & $100.6(34.3-150.3)$ \\
CTD+ILD (169) & $30.0(12.5-61.5)$ & $6.6(3.3-29.4)$ & $139.9(74.6-396.6)$ \\
$P$ & 0.047 & 0.006 & 0.004 \\
\hline
\end{tabular}

Data are presented as medians and IQR. The abbreviations are as in Table 1 and Table 4.

opacity, and cord shadow foci (which often represent non-serious active and reversible foci and the condition is mild) were found in pSS and RA patients; however, more reticular opacity and honeycombing foci (often represent fibrosis and irreversible damage and the condition is severe) were observed in DM/PM and SSc patients. Therefore, the ILDs differed in incidences, manifestations and severity of ILD based on HRCT evaluations in the evaluated CTDs.

PFT is also often used for judgment of severity of ILD. In current study, more normal PFT, OVD, small airway dysfunction and emphysema instances were observed in pSS and RA groups, however, more RVD, and MVD instances were observed in DM/PM and SSc groups. These findings were consistent with HRCT results: OVD and small airway dysfunction might represent the early stage of ILD and were associated with patching, ground-glass opacity, and cord shadow foci (in pSS and RA groups) in HRCT, whereas RVD might represent the late 
stage of ILD and was associated with reticular opacity or honeycombing foci (in DM/PM and SSc groups). Additionally, more patients with DFI were diagnosed by PFT in pSS and SSc groups, which might be due to enhanced emphysema in pSS group and enhanced pulmonary fibrosis in SSc group.

ESR, CRP and SF are often regarded as inflammatory markers. In current study, the levels of ESR, CRP and SF were significantly higher in total CTD+ILD group than in total CTD-ILD group. When patients of every CTD type were classified into CTD+ILD and CTD-ILD groups, the same results were obtained with respect to ESR, CRP and SF levels in different CTDs. Lee SH, et al. reported that increased CRP levels at diagnosis were associated with poor survival in idiopathic pulmonary fibrosis cases [11]. Based on these results, inflammation might be main contributor to CTD+ILD. However, we found that the levels of ESR and CRP were lower in the groups of more serious condition (DM/PM and SSc groups) than in the groups of less serious condition (pSS and RA groups). Moreover, Cavagna L, et al. reported that CRP alleviates neutrophil-induced alveolitis [12] [13]. Therefore, it appears that lower inflammation levels are associated with the higher incidence and worse condition of ILD. How can these contrary conclusions be explained? It has been reported that the focal inflammation of lung results in the development of ILD, therefore, the higher ESR and CRP levels found in RA and pSS groups might mainly represent systemic inflammation, and the levels of ESR and CRP might be higher in lungs of patients with DM/PM and SSc than in lungs of patients with pSS and RA although the levels in serum were lower than in serum of patients with pSS and RA, which needs further confirmation by study of bronchoalveolar lavage fluid (BALF) and lung tissue in future.

The activated phosphoinositide 3-kinase (PI3K)/Akt pathway promotes the repair and development of ILD [14], and CRP promotes PI3K-Akt pathway activation [15], so CRP might contributes to ILD by this pathway. Damage and inflammation are more apparent during the early stage of ILD (nodules, patching and ground-glass opacity), whereas repair is more apparent during the late stage (reticular opacity and honeycombing foci). Therefore, repair might be more apparent in patients with DM/PM or SSc than in patients with RA or pSS. Some researchers reported apparent repair in IPF [16] [17]. In addition, repair markers were found in an SSc model and in patients with SSc [18], and ILD in SSc is similar to IPF [19] [20]. Those results support more repair in SSc-ILD. Interestingly, extracellular ferritin also activates the PI3K pathway [21], possibly representing the causative contribution of SF to ILD in CTDs.

Some of auto-antibodies in serum are associated with CTD+ILD. AECA and anti-SSA were associated with the advent and severity of ILD in DM/PM [9] [22]. Here, we found a significantly higher frequency of anti-SSA in DM/PM+ILD group than in DM/PM-ILD group but not in other CTDs, which indicates that anti-SSA might be a contributor to DM/PM+ILD. We did not find significant correlation between ANA, AECA and CTD+ILDs.

The means of age in pSS+ILD, DM/PM+ILD and total CTD+ILD groups were 
significantly higher than the comparable CTD-ILD groups, so, age may be contribute to the development of ILD in CTDs. However, the higher means of age were in pSS and RA groups, and the lower ones were in DM/PM and SSc groups, and RA group had the highest mean age and the longest disease duration, which indicates that age and disease duration may be not contributors to the higher incidences and more severe condition of ILD in DM/PM and SSc groups than in RA and pSS groups. Additionally, disease duration did not significantly differ between CTD+ILD groups and the comparable CTD-ILD groups, which indicates that disease duration might not be key contributor to the development of ILD in CTDs.

Glucocorticoids and some immunosuppressive agents ameliorate or slow the progression of pathogenetic conditions associated with ILD [23] [24] [25] [26]. However, we didn't find treatment was related to ILDs in different CTDs.

The sample number was relatively small in current study, especially for patients with CTD-ILD, which causes it difficult to analyze the correlation between the antibodies with lower positive incidences and ILD, such as antibodies against aminoacyl-tRNA synthetase to DM/PM+ILD. It needs be studied in larger population in future.

\section{Conclusion}

In summary, there were higher incidences and more severe condition of ILD for $\mathrm{DM} / \mathrm{PM}$ and SSc patients than those for pSS and RA patients, more normal lung HRCTs, nodules, patching, ground-glass opacity, and cord shadow foci in pSS and RA patients, and more reticular opacity and honeycombing foci in DM/PM and SSc patients based on HRCT and PFT evaluations in current study. When excluding the influences of disease duration, and ESR, CRP, SF, age and anti-SSA are positively correlated with ILD in different CTDs. Our results provide important evidences for judging disease condition and prognosis in clinic.

\section{References}

[1] Fischer, A. (2009) Interstitial Lung Disease: A Rheumatologist's Perspective. Journal of Clinical Rheumatology, 15, 95-99. https://doi.org/10.1097/RHU.0b013e31819b715d

[2] Liu, X., Mayes, M.D., Pedroza, C., Draeger, H.T., Gonzalez, E.B., Harper, B.E., et al. (2013) Does C-Reactive Protein Predict the Long-Term Progression of Interstitial Lung Disease and Survival in Patients with Early Systemic Sclerosis? Arthritis Care \& Research, 65, 1375-1380. https://doi.org/10.1002/acr.21968

[3] Muangchan, C., Harding, S., Khimdas, S., Bonner, A., Canadian Scleroderma Research Group, Baron, M. and Pope, J. (2012) Association of C-Reactive Protein with High Disease Activity in Systemic Sclerosis: Results from the Canadian Scleroderma Research Group. Arthritis Care \& Research, 64, 1405-1414. https://doi.org/10.1002/acr.21716

[4] Gono, T., Sato, S., Kawaguchi, Y., Kuwana, M., Hanaoka, M., Katsumata, Y., et al. (2012) Anti-MDA5 Antibody, Ferritin and IL-18 Are Useful for the Evaluation of Response to Treatment in Interstitial Lung Disease with Anti-MDA5 Antibo- 
dy-Positive Dermatomyositis. Rheumatology (Oxford), 51, 1563-1570. https://doi.org/10.1093/rheumatology/kes102

[5] Mimori, T., Nakashima, R. and Hosono, Y. (2012) Interstitial Lung Disease in Myositis: Clinical Subsets, Biomarkers, and Treatment. Current Rheumatology Reports, 14, 264-274. https://doi.org/10.1007/s11926-012-0246-6

[6] D'cruz, D., Keser, G., Khamashta, M.A., Direskeneli, H., Targoff, I.N., Miller, F., et al. (2000) Antiendothelial Cell Antibodies in Inflammatory Myopathies: Distribution among Clinical and Serologic Groups and Association with Interstitial Lung Disease. The Journal of Rheumatology, 27, 161-164.

[7] Jiang, Z., Tao, J.H., Zuo, T., Li, X.M., Wang, G.S., Fang, X., et al. (2016) The Correlation between miR-200c and the Severity of Interstitial Lung Disease Associated with Different Connective Tissue Diseases. Scandinavian Journal of Rheumatology, 46, 122-129. https://doi.org/10.3109/03009742.2016.1167950

[8] Austin, J.H.M., Müller, N.L., Friedman, P.J., Hansell, D.M., Naidich, D.P., Remy-Jardin, M., et al. (1996) Glossary of Terms for CT of the Lungs: Recommendations of the Nomenclature Committee of the Fleischner Society. Radiology, 200, 327-331. https://doi.org/10.1148/radiology.200.2.8685321

[9] La Corte, R., Lo Mo Naco, A., Locaputo, A., Dolzani, F. and Trotta, F. (2006) In Patients with Antisynthetase Syndrome the Occurrence of Anti-Ro/SSA Antibodies Causes a More Severe Interstitial Lung Disease. Autoimmun, 39, 249-253. https://doi.org/10.1080/08916930600623791

[10] Ferri, C., Valentini, G., Cozzi, F., Sebastiani, M., Michelassi, C., La Montagna, G., et al. (2002) Systemic Sclerosis: Demographic, Clinical, and Serologic Features and Survival in 1,012 Italian Patients. Medicine, 81, 139-153. https://doi.org/10.1097/00005792-200203000-00004

[11] Lee, S.H., Shim, H.S., Cho, S.H., Kim, S.Y., Lee, S.K., Son, J.Y., et al. (2011) Prognostic Factors for Idiopathic Pulmonary Fibrosis: Clinical, Physiologic, Pathologic, and Molecular Aspects. Sarcoidosis, Vasculitis, and Diffuse Lung Diseases, 28, 102-112.

[12] Heuertz, R.M., Ahmed, N. and Webster, R.O. (1996) Peptides Derived from C-Reactive Protein Inhibit Neutrophil Alveolitis. Journal of Immunology, 156, 3412-3417.

[13] Zouki, C., Beauchamp, M., Baron, C. and Filep, J.G. (1997) Prevention of in Vitro Neutrophil Adhesion to Endothelial Cells through Shedding of L-Selectin by C-Reactive Protein and Peptides Derived from C-Reactive Protein. Journal of Clinical Investigation, 100, 522-529. https://doi.org/10.1172/JCI119561

[14] Cantley, L.C. (2002) The Phosphoinositide 3-Kinase Pathway. Science, 296, 1655-1657. https://doi.org/10.1126/science.296.5573.1655

[15] Zhong, W., Zen, Q., Tebo, J., Schlottmann, K., Coggeshall, M. and Mortensen, R.F. (1998) Effect of Human C-Reactive Protein on Chemokine and Chemotactic Factor-Induced Neutrophil Chemotaxis and Signaling. Journal of Immunology, 161, 2533-2540.

[16] Margaritopoulos, G.A., Romagnoli, M., Poletti, V., Siafakas, N.M., Wells, A.U. and Antoniou, K.M. (2012) Recent Advances in the Pathogenesis and Clinical Evaluation of Pulmonary Fibrosis. European Respiratory Review, 21, 48-56. https://doi.org/10.1183/09059180.00007611

[17] Hardie, W.D., Glasser, S.W. and Hagood, J.S. (2009) Emerging Concepts in the Pathogenesis of Lung Fibrosis. The American Journal of Pathology, 175, 3-16. https://doi.org/10.2353/ajpath.2009.081170 
[18] Liu, G., Friggeri, A., Yang, Y., Milosevic, J., Ding, Q., Thannickal, V.J., et al. (2010) miR-21 Mediates Fibrogenic Activation of Pulmonary Fibroblasts and Lung Fibrosis. Journal of Experimental Medicine, 207, 1589-1597. https://doi.org/10.1084/jem.20100035

[19] Murray, L.A., Rubinowitz, A. and Herzog, E.L. (2012) Interstitial Lung Disease: Is Interstitial Lung Disease the Same as Scleroderma Lung Disease? Current Opinion in Rheumatology, 24, 656-662. https://doi.org/10.1097/BOR.0b013e3283588de4

[20] Bagnato, G., Roberts, W.N., Roman, J. and Gangemi, S. (2017) A Systematic Review of Overlapping microRNA Patterns in Systemic Sclerosis and Idiopathic Pulmonary Fibrosis. European Respiratory Review, 26, 160125. https://doi.org/10.1183/16000617.0125-2016

[21] Ruddell, R.G., Hoang-Le, D., Barwood, J.M., Rutherford, P.S., Piva, T.J., Watters, D.J., et al. (2009) Ferritin Functions as a Proinflammatory Cytokine via Iron-Independent Protein Kinase C Zeta/Nuclear Factor KappaB-Regulated Signaling in Rat Hepatic Stellate Cells. Hepatology, 49, 887-900.

https://doi.org/10.1002/hep.22716

[22] Váncsa, A., Csípo, I., Németh, J., Dévényi, K., Gergely, L. and Dankó, K. (2009) Characteristics of Interstitial Lung Disease in SS-A Positive/jo-1 Positive Inflammatory Myopathy Patients. Rheumatology International, 29, 989-994.

https://doi.org/10.1007/s00296-009-0884-9

[23] Cavagna, L., Caporali, R., Abdì-Alì, L., Dore, R., Meloni, F. and Montecucco, C. (2013) Cyclosporine in Anti-Jo1-Positive Patients with Corticosteroid-Refractory Interstitial Lung Disease. The Journal of Rheumatology, 40, 484-492. https://doi.org/10.3899/jrheum.121026

[24] Mimori, T., Nakashima, R. and Hosono, Y. (2012) Interstitial Lung Disease in Myositis: Clinical Subsets, Biomarkers, and Treatment. Current Rheumatology Reports, 14, 264-274. https://doi.org/10.1007/s11926-012-0246-6

[25] Pérez Campos, D., Estévez Del Toro, M., Peña Casanovas, A., González Rojas, P.P., Morales Sánchez, L. and Gutiérrez Rojas, A.R. (2012) Are High Doses of Prednisone Necessary for Treatment of Interstitial Lung Disease in Systemic Sclerosis? Reumatología Clínica, 8, 58-62. https://doi.org/10.1016/j.reuma.2011.11.006

[26] Khanna, D., Saggar, R., Mayes, M.D., Abtin, F., Clement, P.J., Maranian, P., et al. (2011) A One-Year, Phase I/IIa, Open-Label Pilot Trial of Imatinib Mesylate in the Treatment of Systemic Sclerosis-Associated Active Interstitial Lung Disease. Arthritis \& Rheumatology, 63, 3540-3546. https://doi.org/10.1002/art.30548 


\section{Supplement}

Table S1. Comparison of age (year) between different CTD-ILD groups and comparable CTD +ILD groups.

\begin{tabular}{cccccc}
\hline & pSS (10/45) & RA (19/39) & DM/PM (6/52) & SSc (1/31) & Total CTD (27/169) \\
\hline CTD-ILD & $43.2 \pm 12.7$ & $46.9 \pm 11.6$ & $34.6 \pm 16.2$ & $36.8 \pm 11.6$ & $42.0 \pm 13.5$ \\
CTD+ILD & $53.1 \pm 11.6$ & $54.0 \pm 14.1$ & $45.5 \pm 14.8$ & $44.0 \pm 13.8$ & $48.8 \pm 14.3$ \\
$P$ value & 0.003 & 0.089 & 0.039 & 0.273 & 0.002 \\
\hline
\end{tabular}

Data are presented as means $\pm \mathrm{SD}$. The numbers in round brackets after every CTD represent the numbers of CTD-ILD and CTD+ILD patients. Abbreviations: pSS: primary Sjogren's symptom, RA: rheumatoid arthritis, DM/PM: dermatomyositis/polymyositis, SSc: systemic sclerosis, CTD-ILD: connective tissue disease without interstitial lung disease, CTD+ILD: CTD with ILD.

Table S2. Comparison of disease duration (month) between different CTD-ILD groups and comparable CTD+ILD groups.

\begin{tabular}{cccccc}
\hline & $\mathrm{pSS}(10 / 45)$ & $\mathrm{RA}(19 / 39)$ & $\mathrm{DM} / \mathrm{PM}(6 / 52)$ & $\mathrm{SSc}(1 / 31)$ & $\begin{array}{c}\text { Total } \\
\mathrm{CTD}(27 / 169)\end{array}$ \\
\hline CTD-ILD & 48.0 & 48.0 & 24.0 & 24.0 & 48.0 \\
& $(27.0-84.0)$ & $(12.0-123.0)$ & $(6.0-48.0)$ & $(15.5-78.0)$ & $(12.0-84.0)$ \\
CTD+ILD & 60.0 & 84.0 & 12.0 & 48.0 & 48.0 \\
& $(12.0-120.0)$ & $(18.0-213.0)$ & $(4.0-60.0)$ & $(24.0-96)$ & $(12.0-105.0)$ \\
$P$ value & 0.985 & 0.269 & 0.734 & 0.380 & 0.876 \\
\hline
\end{tabular}

Data are presented as medians and IQR. The numbers in round brackets after every CTD name represent the numbers of CTD-ILD and CTD+ILD patients. Abbreviations: as in Table 1.

Table S3. Treatment protocols for the past three months (No./\%).

\begin{tabular}{ccccc}
\hline & pSS (57) & RA (52) & DM/PM (59) & SSc (41) \\
\hline Hydroxychloroquine Sulfate & $19(33.3)$ & $8(15.4)$ & $19(32.2)$ & $4(9.8)$ \\
Mycophenolate mofetil & $3(5.3)$ & 0 & $2(3.4)$ & $2(4.9)$ \\
Methotrexate & $3(5.3)$ & $15(28.8)$ & $8(13.6)$ & $4(9.8)$ \\
Leflunomide & $1(1.8)$ & $13(25.0)$ & 0 & $3(7.3)$ \\
Cyclophosphamide & $1(1.8)$ & $1(1.9)$ & $5(8.5)$ & $7(17.1)$ \\
Penicillamine & $1(1.8)$ & 0 & $1(1.7)$ & $19(46.3)$ \\
Thalidomide & 0 & $3(5.7)$ & $8(13.6)$ & $4(9.8)$ \\
Etanercept & 0 & $2(3.8)$ & 0 & 0 \\
Azathioprine & 0 & $2(3.8)$ & $5(8.5)$ & $3(7.3)$ \\
Intravenous immunoglobulin & 0 & 0 & $3(5.1)$ & 0 \\
Tacrolimus & 0 & 0 & $1(1.7)$ & $1(2.4)$ \\
\hline
\end{tabular}

Data are presented as number and percentage. Abbreviations: as in Table 1. 